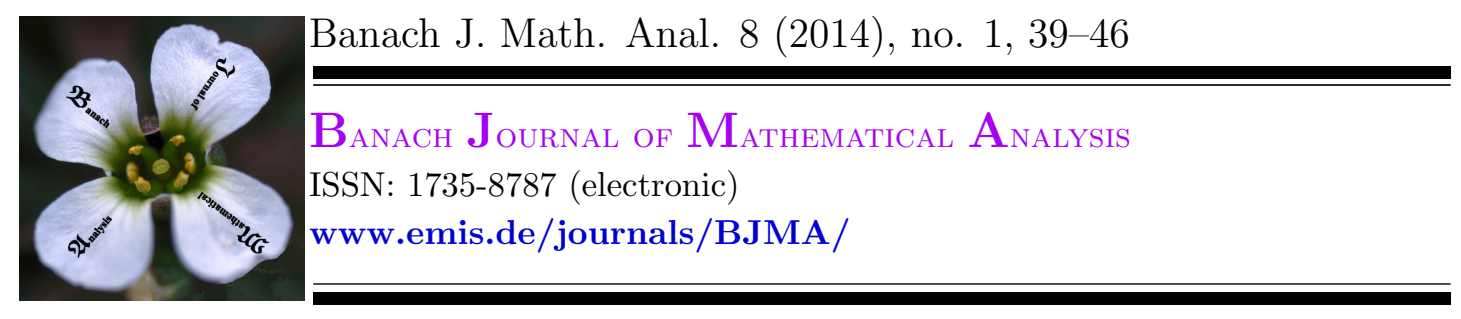

\title{
LINEAR MAPS BETWEEN OPERATOR ALGEBRAS PRESERVING CERTAIN SPECTRAL FUNCTIONS
}

\author{
XIAOHONG CAO* AND SHIZHAO CHEN \\ Communicated by A. R. Villena
}

\begin{abstract}
Let $H$ be an infinite dimensional complex Hilbert space and let $\phi$ be a surjective linear map on $B(H)$ with $\phi(I)-I \in \mathcal{K}(H)$, where $\mathcal{K}(H)$ denotes the closed ideal of all compact operators on $H$. If $\phi$ preserves the set of upper semi-Weyl operators and the set of all normal eigenvalues in both directions, then $\phi$ is an automorphism of the algebra $B(H)$. Also the relation between the linear maps preserving the set of upper semi-Weyl operators and the linear maps preserving the set of left invertible operators is considered.
\end{abstract}

\section{INTRODUCTION AND PRELIMINARIES}

Let $H$ be an infinite dimensional complex Hilbert space and $B(H)$ the algebra of all bounded linear operators on $H$ and $\mathcal{K}(H) \subseteq B(H)$ be the closed ideal of all compact operators. We write $T^{*}$ for the conjugate operator of $T \in B(H)$. An operator $T \in B(H)$ is called upper semi-Fredholm if it has closed range $R(T)$ with finite dimensional null space $N(T)$ and if $R(T)$ has finite co-dimension, $T \in B(H)$ is called a lower semi-Fredholm operator. We call $T \in B(H)$ Fredholm if it has closed range with finite dimensional null space and its range of finite co-dimension. For a semi-Fredholm operator $T \in B(H)$ (upper semi-Fredholm operator or lower semi-Fredholm operator), let $n(T)=\operatorname{dim} N(T)$ and $d(T)=$ $\operatorname{dim} H / R(T)=\operatorname{codim} R(T)$. The index of a semi-Fredholm operator $T \in B(H)$ is given by $i n d(T)=n(T)-d(T)$. The operator $T$ is Weyl if it is Fredholm of index

Date: Received: 14 December 2012; Revised: 20 February 2013; Accepted: 7 March 2013.

* Corresponding author.

2010 Mathematics Subject Classification. Primary 47B48; Secondary 47A10, 46H05.

Key words and phrases. Calkin algebra, upper semi-Weyl operator, linear preservers, left invertible. 
zero; $T$ is called Browder if $T$ is Fredholm with finite ascent and finite descent; $T \in B(H)$ is called upper semi-Weyl if $T$ is upper semi-Fredholm with $\operatorname{ind}(T) \leq 0$. Let $S F_{+}^{-}(H)$ denote the set of all upper semi-Weyl operators and let $\sigma_{e a}(T)=$ $\left\{\lambda \in \mathbb{C}: T-\lambda I \notin S F_{+}^{-}(H)\right\}$ be the essential approximate point spectrum of T. $\sigma(T), \sigma_{e}(T), \sigma_{S F_{+}}(T), \sigma_{S F_{-}}(T), \sigma_{w}(T)$ and $\sigma_{b}(T)$ denote the spectrum, the essential spectrum, the upper semi-Fredholm spectrum, the lower semi-Fredholm spectrum, the Weyl spectrum and the Browder spectrum respectively $([8,9])$. Let $\sigma_{0}(T)=\sigma(T) \backslash \sigma_{b}(T)$ denote the set of all normal eigenvalues.

Let $\Phi(H) \subseteq B(H)$ be the set of all Fredholm operators. We denote the Calkin algebra $B(H) / \mathcal{K}(H)$ by $\mathcal{C}(H)$. Let $\pi: B(H) \rightarrow \mathcal{C}(H)$ be the quotient map. A bijective linear map $\phi: B(H) \rightarrow B(H)$ is called a Jordan isomorphism if $\phi\left(A^{2}\right)=(\phi(A))^{2}$ for every $A \in B(H)$, or equivalently $\phi(A B+B A)=\phi(A) \phi(B)+$ $\phi(B) \phi(A)$ for all $A$ and $B$ in $B(H)$. It is obvious that every isomorphism and every anti-isomorphism is a Jordan isomorphism. For further properties of Jordan homomorphisms, we refer the reader to [10] and [11].

In the last two decades there has been considerable interest in the so-called linear preserver problems (see [1, 5, 16]). The goal of studying linear preservers is to give structural characterizations of linear maps on algebras having some special properties such as leaving invariant a certain subset of the algebra, or leaving invariant a certain function on the algebra. One of the most famous problem in this direction is Kaplansky's problem([13]): Let $\phi$ be a surjective linear map between two semi-simple Banach algebras $\mathcal{A}$ and $\mathcal{B}$. Suppose that $\sigma(\phi(x))=\sigma(x)$ for all $x \in \mathcal{A}$. Is it true that $\phi$ is Jordan isomorphism? This problem was first solved in the finite dimensional case. J.Dieudonně ([7]) and Marcus and Purves ([15]) proved that every unital invertibility preserving linear map on a complex matrix algebra is either an inner automorphism or a linear antiautomorphism. This result was later extended to the algebra of all bounded linear operators on a Banach space by A.R.Sourour([22]) and to von Neumann algebra by B.Aupetit([1]). Many other linear preserver problems have been extended to the infinite dimensional case. For the most significant partial obtained in this direction, we refer the reader to $([1,18,22,23])$. New contributions to the study of linear preserver problem in $B(H)$ have been recently made by Mbekhta in [17], Mbekhta, Rodman and Šemrl in [18], Mbekhta and Šemrl in [16] and Bendaoud, Bourhim and Sarih in [4].

In this article, we give the characterization of automorphism on $B(H)$. We get that: Let $\phi$ be a surjective linear maps on $B(H)$ with $\phi(I)-I \in \mathcal{K}(H)$ preserving the set of upper semi-Weyl operators and the set of all normal eigenvalues in both directions, then $\phi$ is an automorphism of the algebra $B(H)$. Also the relation between the linear maps preserving the set of upper semi-Weyl operators and the linear maps preserving the set of left invertible operators is considered.

\section{MAin RESUlts}

An operator is left invertible if it has a left inverse. It turns out that an operator $T \in B(H)$ is left invertible if and only if it is bounded below, or equivalently, it is upper semi-Fredholm with $n(T)=0$. Let $\sigma_{a}(T)=\{\lambda \in \mathbb{C}: T-\lambda I$ is 
not left invertible\}. We say that a linear map $\phi: B(H) \rightarrow B(H)$ preserves the set of upper semi-Weyl operators (left invertible operators) in both directions if $T \in S F_{+}^{-}(H)(T$ is left invertible $) \Leftrightarrow \phi(T) \in S F_{+}^{-}(H)(\phi(T)$ is left invertible).

A linear map $\phi: B(H) \rightarrow B(H)$ is said to be surjective up to compact operators if for every $T \in B(H)$ there exists $T^{\prime} \in B(H)$ such that $T-\phi\left(T^{\prime}\right) \in \mathcal{K}(H)$. It is clear that if $\phi$ is surjective, then it is surjective up to compact operators.

Remark 2.1. (1) If a linear map $\phi: B(H) \rightarrow B(H)$ preserves the set of upper semi-Weyl operators in both directions, we can not induce that $\phi$ preserves the set of left invertible operators in both directions. For example, let $A, B \in B\left(\ell_{2}\right)$ be defined by:

$$
\begin{gathered}
A\left(x_{1}, x_{2}, x_{3}, \cdots\right)=\left(x_{2}, x_{3}, \cdots\right), \\
B\left(x_{1}, x_{2}, x_{3}, \cdots\right)=\left(0, x_{1}, x_{2}, x_{3}, \cdots\right),
\end{gathered}
$$

and let $\phi(T)=A T B, T \in B\left(\ell_{2}\right)$. We can see that both $A$ and $B$ are Fredholm operators, and $i n d(A)+i n d(B)=0$. By the properties of the index it follows that $T \in S F_{+}^{-}\left(B\left(\ell_{2}\right)\right)$ if and only if $\phi(T) \in S F_{+}^{-}\left(B\left(\ell_{2}\right)\right)$. For any $T \in B\left(\ell_{2}\right)$, let $T_{1}=B T A$, then $\phi\left(T_{1}\right)=T$. Thus $\phi: B\left(\ell_{2}\right) \rightarrow B\left(\ell_{2}\right)$ is surjective and $\phi$ preserves the set of upper semi-Weyl operators in both directions. But $\phi$ does not preserve the set of left invertible operators in both directions. In fact, for an operator $T \in B\left(\ell_{2}\right)$ defined by:

$$
T\left(x_{1}, x_{2}, x_{3}, \cdots\right)=\left(x_{2}-x_{1}, x_{2}-x_{1}, x_{3}, x_{4} \cdots\right),
$$

we can find that $\phi(T)=I$ is left invertible but $T$ is not left invertible.

(2) If a linear map $\phi: B(H) \rightarrow B(H)$ preserves the set of left invertible operators in both directions, we can not induce that $\phi$ preserves the set of upper semi-Weyl operators in both directions. For example, let $A \in B\left(\ell_{2}\right)$ be defined by:

$$
A\left(x_{1}, x_{2}, x_{3}, \cdots\right)=\left(0,0, x_{1}, x_{2}, \cdots\right),
$$

$B \in B\left(\ell_{2}\right)$ is invertible and let $\phi(T)=A T B, T \in B\left(\ell_{2}\right)$. We can see that $A$ is left invertible, there exists $A_{1} \in B\left(\ell_{2}\right)$ such that $A_{1} A=I$. Since $A \in B\left(\ell_{2}\right)$ is Fredholm, there are $A_{2} \in B\left(\ell_{2}\right)$ and a compact operator $K_{0}$ satisfying $A A_{2}=$ $I+K_{0}$. For any $T \in B\left(\ell_{2}\right)$, let $T_{0}=A_{2} T B^{-1}$ and $K=-K_{0} T$. Then $K$ is compact and $T=\phi\left(T_{0}\right)+K$, which means that $\phi$ is surjective up to compact operators. For any left invertible operator $T \in B\left(\ell_{2}\right)$, suppose that $T_{1} T=I$. Then $B^{-1} T_{1} A_{1} \phi(T)=I$, this shows that $\phi(T)$ is left invertible. For the converse, if $\phi(T)$ is left invertible and suppose $D \phi(T)=I$. Then $B D A T=B D A T B B^{-1}=$ $B D \phi(T) B^{-1}=B B^{-1}=I$, thus $T \in B\left(\ell_{2}\right)$ is left invertible. It follows that $\phi$ preserves the set of left invertible operators in both directions. But $\phi$ does not preserve the set of upper semi-Weyl operators in both directions. In fact, let $T \in B\left(\ell_{2}\right)$ be defined as $T\left(x_{1}, x_{2}, x_{3}, \cdots\right)=\left(x_{2}, x_{3}, \cdots\right)$, then $\phi(T)$ is upper semi-Weyl with $\operatorname{ind}(\phi(T))=\operatorname{ind}(A)+\operatorname{ind}(T)+\operatorname{ind}(B)=-2+1+0=-2$ but $T$ is not upper semi-Weyl.

It is well known that the set of left invertible operators is a subset of $S F_{+}^{-}(H)$, we need to study the relation between the linear maps preserving the set of upper semi-Weyl operators and the linear maps preserving the set of left invertible operators. Let's begin with a Theorem. 
Theorem 2.2. Let $\phi: B(H) \rightarrow B(H)$ be a surjective linear map preserving upper semi-Weyl operators in both directions and $\phi(I)-I \in \mathcal{K}(H)$. If $\sigma_{0}(K)=$ $\sigma_{0}(\phi(K))$ for any Riesz operator $K$, then there is an invertible linear operator $A \in B(H)$ such that $\phi(T)=A T A^{-1}$ for any $T \in B(H)$.

Proof. We will prove the Theorem by seven steps:

(i) For any $T \in B(H), \sigma_{e a}(T)=\sigma_{e a}(\phi(T))$.

Let $\phi(I)=I+K$, where $K \in \mathcal{K}(H)$. Since $T-\lambda I \in S F_{+}^{-}(H) \Leftrightarrow \phi(T-\lambda I)=$ $\phi(T)-\lambda \phi(I)=\phi(T)-\lambda I-\lambda K \in S F_{+}^{-}(H) \Leftrightarrow \phi(T)-\lambda I \in S F_{+}^{-}(H)$, it follows that $\sigma_{e a}(T)=\sigma_{e a}(\phi(T))$ for any $T \in B(H)$.

(ii) $\phi$ preserves compact operators in both directions.

First we claim that

$$
\begin{gathered}
\mathcal{K}(H)=\left\{K \in B(H): K+S F_{+}^{-}(H) \in S F_{+}^{-}(H)\right\} \\
=\left\{K \in B(H): \sigma_{e a}(T+K)=\sigma_{e a}(T) \text { for all } T \in B(H)\right\} .
\end{gathered}
$$

From the stability properties of index function, it is clear that $\mathcal{K}(H) \subseteq\{K \in$ $\left.B(H): K+S F_{+}^{-}(H) \in S F_{+}^{-}(H)\right\}=\left\{K \in B(H): \sigma_{e a}(T+K)=\sigma_{e a}(T)\right.$ for all $T \in$ $B(H)\}$.

Let $\partial E$ and $\eta E$ denote the boundary and the polynomial convex hull of a compact subset $E$ of $\mathbb{C}$ respectively. For any $T \in B(H)$, since

$$
\partial \sigma_{w}(T) \subseteq \partial \sigma_{e}(T) \subseteq \sigma_{e}(T) \subseteq \sigma_{w}(T) \text { and } \partial \sigma_{w}(T) \subseteq \partial \sigma_{e a}(T) \subseteq \sigma_{e a}(T) \subseteq \sigma_{w}(T)
$$

it follows that $\eta \sigma_{e a}(T)=\eta \sigma_{w}(T)=\eta \sigma_{e}(T)$.

Now, let $K \in B(H)$ such that $\sigma_{e a}(T+K)=\sigma_{e a}(T)$ for all $T \in B(H)$. Then by Theorem 5.3.1 in [2], $\eta \sigma_{e}(T+K)=\eta \sigma_{e}(T)$ for all $T \in B(H)$. Taking into account the semisimplicity of $\mathcal{C}(H)$ and the spectral characterization of the radical, it is not difficult to prove that the $\mathcal{K}(H)=\left\{K \in B(H): K+S F_{+}^{-}(H) \in S F_{+}^{-}(H)\right\}=$ $\left\{K \in B(H): \sigma_{e a}(T+K)=\sigma_{e a}(T)\right.$ for all $\left.T \in B(H)\right\}$.

Let $K \in \mathcal{K}(H)$, for any $T \in S F_{+}^{-}(H)$, since $\phi$ preserves upper semi-Weyl operators in both directions, there exists $T^{\prime} \in S F_{+}^{-}(H)$ for which $T=\phi\left(T^{\prime}\right)$. Hence $T+\phi(K)=\phi\left(T^{\prime}\right)+\phi(K)=\phi\left(T^{\prime}+K\right) \in S F_{+}^{-}(H)$. Then $\phi(K) \in \mathcal{K}(H)$. For the converse, let $\phi(K) \in \mathcal{K}(H)$, for any $T \in S F_{+}^{-}(H), \phi(T+K)=\phi(T)+$ $\phi(K) \in S F_{+}^{-}(H)$, then $T+K \in S F_{+}^{-}(H)$. It follows that $K \in \mathcal{K}(H)$. Now we prove that $\phi$ preserves compact operators in both directions.

Since $\phi$ preserves compact operators in both directions, it follows that $\sigma(K)=$ $\{0\} \cup \sigma_{0}(K)=\{0\} \cup \sigma_{0}(\phi(K))=\sigma(\phi(K))$ for any compact operator $K$.

(iii) $N(\phi) \subseteq \mathcal{K}(H)$.

If $K \in N(\phi)$ and $T \in S F_{+}^{-}(H)$, then $\phi(T+K)=\phi(T) \in S F_{+}^{-}(H)$. Thus for all $T \in S F_{+}^{-}(H), T+K \in S F_{+}^{-}(H)$. Thus $K \in \mathcal{K}(H)$.

(iv) Let $\varphi: \mathcal{C}(H) \rightarrow \mathcal{C}(H)$ be an induced linear map such that $\phi \circ \pi=\pi \circ \phi$, then $\varphi$ is isomorphism.

$\phi$ induces a linear map $\varphi: \mathcal{C}(H) \rightarrow \mathcal{C}(H)$ such that $\varphi \circ \pi=\pi \circ \phi$. Clearly, $\varphi$ is surjective since $\phi$ is surjective. By hypothesis and (ii), $\varphi$ is $\eta \sigma$-preserving. From Corollary 2.3 in [5], $\varphi$ is injective, and by Theorem 3.1 in [5], $\varphi$ is either a homomorphism or an anti-homomorphism. 
First we will prove that $\phi$ preserves upper semi-Fredholm operators in both directions. By Theorem 2.1 in [17], we know that $\phi$ preserves Fredholm operators in both directions. Let $T \in B(H)$ be an upper semi-Fredholm, there are two cases to consider: $d(T)=\infty$ and $d(T)<\infty$. If $d(T)=\infty$, using the fact that $\phi$ is a linear map preserving upper semi-Weyl operators in both directions, we know that $\phi(T)$ is upper semi-Fredholm. If $d(T)<\infty$, then $T$ is Fredholm, thus $\phi(T)$ is Fredholm since $\phi$ preserves Fredholm operators in both directions. Using the same way, we can prove that $T$ is upper semi-Fredholm if $\phi(T)$ is upper semi-Fredholm. By Corollary 3.6 in [3], $\varphi$ is an isomorphism.

As $\phi$ preserves the essential spectrum, from Theorem 3.3 in [17] we deduce that $\operatorname{ind}(\phi(T))=\operatorname{ind}(T)$ or ind $(\phi(T))=-i n d(T)$ for every Fredholm operator $T \in$ $B(H)$. Since $\phi$ preserves upper semi-Weyl operators in both directions, it follows that $\operatorname{ind}(\phi(T)) \cdot \operatorname{ind}(T) \geq 0$ for any $T \in \Phi(H)$. Thus $\operatorname{ind}(\phi(T))=\operatorname{ind}(T)$ for any $T \in \Phi(H)$. Also we can prove that $i n d(\phi(T))=i n d(T)$ for any upper semiFredholm operator $T \in B(H)$. For lower semi-Fredholm operator $T \in B(H)$, we also have $\operatorname{ind}(\phi(T))=\operatorname{ind}(T)$. In fact, since $\varphi$ is an isomorphism, by Corollary 3.6 in [3], $\phi$ preserves lower semi-Fredholm operators in both directions. Let $T \in B(H)$ be a lower semi-Fredholm operator, then $\phi(T)$ is a lower semi-Fredholm operator. There are also two cases to consider: $n(T)=\infty$ and $n(T)<\infty$. If $n(T)=\infty$, using the fact that $\phi$ is a linear map preserving Fredholm operators in both directions, we know that $n(\phi(T))=\infty$, then $\operatorname{ind}(\phi(T))=\operatorname{ind}(T)=\infty$. If $n(T)<\infty$, then $T$ is Fredholm, thus $\phi(T)$ is Fredholm since $\phi$ preserves Fredholm operators in both directions. Then ind $(\phi(T))=\operatorname{ind}(T)$ again.

(v) $\phi$ is injective.

If $\phi(T)=0$, then $T$ is compact and hence $\sigma(T)=\{0\} \cup \sigma_{0}(T)=\{0\} \cup$ $\sigma_{0}(\phi(T))=\{0\}$ since $\sigma_{0}(\phi(T))=\emptyset$. This means that $T$ is quasinipotent. Assume that $T \neq 0$, we can find $x \in H$ such that $T x=y \neq 0$. Clearly, $x$ and $y$ are linear independent. Define a nilpotent operator $N \in B(H)$ by:

$$
N x=x-y, N y=x-y, N z=0, \text { for } z \in\{x, y\}^{\perp} .
$$

Then both $N$ and $N+T$ are compact, thus $\phi(N+T)=\phi(N)$ is compact. From the condition we can find $\sigma(T+N)=\sigma(\phi(T+N))$, then $\sigma(T+N)=\sigma(\phi(T+N))=$ $\sigma(\phi(N))=\sigma(N)=\{0\}$, which means that $T+N$ is quasinilpotent. This is in contraction to the fact that $1 \in \sigma(T+N)$.

(vi) $\phi(T)$ is an idempotent of rank one if and only if $T$ is an idempotent of rank one.

Let $P \in B(H)$ be an idempotent of rank one and let $\phi(P)=Q$. Since both $P$ and $Q$ are compact operators, $\sigma(Q)=\sigma(P)=\{0,1\}$. For any $K \in F_{2}(H)$, where $F_{2}(H)$ denotes the set of all operators in $B(H)$ with rank not greater than 2, there is $S \in B(H)$ such that $K=\phi(S)$ as $\phi$ is surjective. Thus by Theorem 1 in [12] we must have that $\sigma(S+P) \cap \sigma(S+2 P) \subseteq \sigma(S)$. Since $S+P, S+2 P$ and $S$ are all compact operators, it follows that $\sigma(S+P)=\sigma(\phi(S+P))=\sigma(K+Q)$, $\sigma(S+2 P)=\sigma(\phi(S+2 P))=\sigma(K+2 Q)$ and $\sigma(S)=\sigma(\phi(S))=\sigma(K)$. Then $\sigma(K+Q) \cap \sigma(K+2 Q) \subseteq \sigma(K)$. By Lemma 2.2 in [6], we know that $\operatorname{rank} Q=1$. This implies that $Q$ satisfies a quadratic polynomial equation $p(Q)=0$ ([14]). 
Using the fact that $\sigma(Q)=\{0,1\}$, we know that $p$ is of the form $p(\lambda)=\lambda(\lambda-1)$. Then $Q^{2}=Q$.

We get that $\phi$ preserves idempotent of rank one. The same must be true for $\phi^{-1}$, and consequently, $\phi$ preserves idempotents of rank one in both directions. According to Proposition 2.6 in [19] there exists either an invertible $A \in B(H)$ such that $\phi(T)=A T A^{-1}$ for all finite rank operators $T \in B(H)$, or a bounded invertible conjugate-linear operator $C$ on $H$ such that $\phi(T)=C T^{*} C^{-1}$ for every $T \in B(H)$ of finite rank.

(vii) There is an invertible linear operator $A \in B(H)$ such that $\phi(T)=$ $A T A^{-1}$ for any $T \in B(H)$.

Let $T \in B(H)$ such that $T^{2}=0$. Then $\sigma(T)=\{0\}$ and $\sigma_{0}(T)=\emptyset$. Since $T-\lambda I$ is Weyl for any $\lambda \neq 0$ and $\phi$ is a linear map preserving upper semi-Weyl operators in both directions, it follows that $\phi(T)-\lambda I$ is Weyl for any $\lambda \neq 0$. This implies that $\phi(T)$ is a Riesz operator. For every operator $U$ of rank one, we know that both $T+U$ and $\phi(T)+\phi(U)$ are Riesz operators. Then $\sigma(T+U)=$ $\sigma(\phi(T)+\phi(U))$. By assuming that $\phi(U)=A U A^{-1}$, this can be rewritten as $\sigma(T+U)=\sigma\left(A^{-1} \phi(T) A+U\right)$ for each rank one operator $U$. This gives directly that $T=A^{-1} \phi(T) A$, and hence $\phi(T)=A T A^{-1}$. Then $\phi(T)=A T A^{-1}$ for every $T \in B(H)$ by Theorem 2 in [20].

In the second case we show that similarly that $\phi(T)=C T^{*} C^{-1}$ for all $T \in$ $B(H)$. It follows from that $\operatorname{ind}(T)=\operatorname{ind}(\phi(T))$ if $T$ is Fredholm, we know that the second case cannot occur. The proof of the Theorem is complete.

In the proof of Theorem 2.2, we use P.Šemrl's method in Theorem 4 in [21], but there are many differences in two proofs.

Similar to the proof of Lemma 1 in [12], we can get that: Let $A \in B(H)$. If $\sigma_{a}(T+A) \subseteq \sigma_{a}(T)$ for every rank one operator $T$, then $A=0$.

For surjective linear map $\phi: B(H) \rightarrow B(H)$, if $\sigma_{a}(T) \subseteq \sigma_{a}(\phi(T))$ for any $T \in B(H)$ and $\sigma_{a}(T)=\sigma_{a}(\phi(T))$ for any Riesz operator $T$, then $\phi(I)=I$. In fact, suppose that $\phi(S)=I$. For any rank one operator $F$, since $\sigma_{a}(F+S-I)=$ $\sigma_{a}(F+S)-1 \subseteq \sigma_{a}(\phi(F)+\phi(S))-1=\sigma_{a}(\phi(F)+I)-1=\sigma_{a}(\phi(F))=\sigma_{a}(F)$, we know that $S-I=0$, then $S=I$, which means that $\phi(I)=I$. In the proof of Theorem 2.2, we can see that if $\phi$ preserves Riesz operators in both directions and if $\sigma_{0}(T)=\sigma_{0}(\phi(T))$ for any Riesz operator $T$, then there exists either an invertible $A \in B(H)$ such that $\phi(T)=A T A^{-1}$ for every $T \in B(H)$, or a bounded invertible conjugate-linear operator $C$ on $H$ such that $\phi(T)=C T^{*} C^{-1}$ for every $T \in B(H)$.

Corollary 2.3. Let $\phi: B(H) \rightarrow B(H)$ be a surjective linear map preserving upper semi-Weyl operators in both directions. If $\sigma_{a}(T) \subseteq \sigma_{a}(\phi(T))$ for any $T \in$ $B(H)$ and $\sigma_{a}(T)=\sigma_{a}(\phi(T))$ for any Riesz operator $T$, then there is an invertible linear operator $A \in B(H)$ such that $\phi(T)=A T A^{-1}$ for any $T \in B(H)$.

Proof. Since $\phi(I)=I$ and $\phi: B(H) \rightarrow B(H)$ preserves upper semi-Weyl operators in both directions, we can prove that $\phi$ preserves Riesz operators in both directions. Then $\sigma(T)=\sigma_{a}(T)=\sigma_{a}(\phi(T))=\sigma(\phi(T))$ for any Riesz operator $T$. 
Thus $\sigma_{0}(T)=\sigma_{0}(\phi(T))$ for any Riesz operator $T$. By Theorem 2.2, the result is true.

Corollary 2.4. Let $\phi: B(H) \rightarrow B(H)$ be a surjective linear map. If $\phi(I)-I \in$ $\mathcal{K}(H)$ and $\sigma_{0}(T)=\sigma_{0}(\phi(T))$ for any Riesz operator $T \in B(H)$, then the following statements are equivalent:

(1) $\sigma_{a}(T)=\sigma_{a}(\phi(T))$ for any $T \in B(H)$;

(2) $\sigma_{e a}(T)=\sigma_{e a}(\phi(T))$ for any $T \in B(H)$;

(3) $\sigma_{e}(T)=\sigma_{e}(\phi(T))$ and $\operatorname{ind}(T)=$ ind $(\phi(T))$ if $T$ is a Fredholm operator;

(4) $\sigma_{S F_{+}}(T)=\sigma_{S F_{+}}(\phi(T))$ and $i n d(T)=i n d(\phi(T))$ if $T$ is an upper semiFredholm operator;

(5) $\sigma_{S F_{-}}(T)=\sigma_{S F_{-}}(\phi(T))$ and $\operatorname{ind}(T)=\operatorname{ind}(\phi(T))$ if $T$ is a lower semiFredholm operator;

(6) There exists an invertible operator $A \in B(H)$ such that $\phi(T)=A T A^{-1}$ for every $T \in B(H)$.

Proof. It follows from Theorem 2.2, Theorem 2.1 in [17], Theorem 4.8 in [3] and Corollary 3.6 in [3], that (2), (3), (4), (5) and (6) are equivalent. The implication $(6) \Rightarrow(1)$ is clear, and the converse can be argued as in Theorem 4 in [21].

From the proof of Theorem 4 in [21], we know that if $\phi: B(H) \rightarrow B(H)$ be a surjective linear map and $\sigma_{a}(T)=\sigma_{a}(\phi(T))$ for any $T \in B(H)$, then (2), (3), (4) and (5) in Corollary 2.4 are true.

Remark 2.5. In Corollary 2.4, the condition " $\sigma_{0}(T)=\sigma_{0}(\phi(T))$ for any Riesz operator $T \in B(H)$ " is essential. For example, let $A, B \in B\left(\ell_{2}\right)$ and $\phi$ be defined as in (1) in Remark 2.1. Then $\phi: B(H) \rightarrow B(H)$ is a surjective linear map preserving upper semi-Weyl operators in both directions and $\phi(I)=I$, which means that $\sigma_{e a}(T)=\sigma_{e a}(\phi(T)$ ) for any $T \in B(H)$ (from the proof of Theorem 2.2). Let $T_{0}=B A$, then $T_{0}\left(x_{1}, x_{2}, x_{3}, \cdots\right)=\left(0, x_{2}, x_{3}, x_{4}, \cdots\right)$ and $\phi\left(T_{0}\right)=I$. Since $T_{0}=T_{0}^{2}$ and $\phi\left(T_{0}\right)$ is invertible, we can see that $0 \in \sigma_{0}\left(T_{0}\right)$ but $0 \notin \sigma_{0}\left(\phi\left(T_{0}\right)\right)$. Then we can not induce that $\phi$ preserves the set of left invertible operators in both directions from (1) in Remark 2.1.

Acknowledgement. We are grateful to the referees for helpful comments concerning this paper. This work is supported by the Innovation Funds of Graduate Programs, Shaanxi Normal University (No.2013CXS023).

\section{REFERENCES}

1. B. Aupetit, Spectrum-preserving linear mapping between Banach algebra or Jordan Banach algebras, J. London Math. Soc. 62 (2000), 917-924.

2. B. Aupetit, A Primer on spectral theory, Springer-Verlag, 1990.

3. M. Bendaoud, A. Bourhim, M. Burgos and M. Sarih, Linear maps preservig Fredholm and Atkinson elements of $C^{*}$-algebra, Linear Multilinear Algebra 57 (2009), no. 8, 823-838.

4. M. Bendaoud, A. Bourhim and M. Sarih, Linear maps preservig the essential spectral radius, Linear Algebra Appl. 428 (2008), 1041-1045.

5. J. Cui and J. Hou, Linear maps between Banach algebras compressing certain spectral functions, Rocky Mountain J. Math. 34 (2004), no. 2, 565-585.

6. J. Cui and J. Hou, Additive maps on standard operator algebras preserving parts of the spectrum, J. Math. Anal. Appl. 282 (2003), 266-278. 
7. J. Dieudonně, Sur une généralisation du groupe orthogonal à quatre variables, Arch. Math. (Basel) 1 (1949), 282-287.

8. R.E. Harte, Invertibility and singularity for bounded linear operators, Dekker, New York, 1988.

9. R.E. Harte, Fredholm, Weyl and Browder theory, Proc. Royal Irish Acad. 85 (1985), A(2), 151-176.

10. I.N. Herstein, Jordan homomorphisms, Trans. Amer. Math. Soc. 81 (1956), 331-341.

11. N. Jacobson and C.E. Rickart, Jordan homomorphisms of rings, Trans. Amer. Math. Soc. 69 (1950), 479-502.

12. A.A. Jafarian and A.R. Sourour, Spectrum-preserving linear maps, J. Funct. Anal. 66 (1986), 255-261.

13. I. Kaplansky, Algebraic and analytic aspects of opertors algebras, Amer. Math. Soc. Providence, 1970.

14. I. Kaplansky, Infinite Abelian Groups, University of Michigan Press, Ann Arbor, 1954.

15. M. Marcus and R. Purves, Linear transformations on algebras of matrices: The invariance of the elementary symmetric functions, Canad. J. Math. 11 (1959), 383-396.

16. M. Mbekhta and P. Šemrl, Linear maps preserving semi-Fredholm operators and generalized invertibility, Linear Multilinear Algebra 57 (2009), 55-64.

17. M. Mbekhta, Linear maps preserving the set of Fredholm operators, Proc. Amer. Math. Soc. 135 (2007), no. 11, 3613-3619.

18. M. Mbekhta, L. Rodman and P.Šemrl, Linear maps preserving generalized invertibility, Integral Equations Operator Theory 55 (2006), 93-109.

19. M. Omladič, On operators preserving commutativity, J. Funct. Anal. 66 (1986), 105-122.

20. C. Pearcy and D.Topping, Sums of small number of idempotents, Michigan Math. J. 14 (1967), 453-465.

21. P. Šemrl, Two characterizations of automorphisms on B(X), Studia Math. 105 (1993), no. 2, 143-149.

22. A.R. Sourour, Invertibility preserving linear maps on $L(H)$, Trans. Amer. Math. Soc. 348 (1996), no. 1, 13-30.

23. W. Żelazko, A characterization of multiplicative linear functionals in complex Banach algebras, Studia Math. 30 (1968), 83-85.

College of Mathematics and Information Science, ShaAnxi Normal University, Xi'An, 710062, People's Republic of China.

E-mail address: xiaohongcao@snnu.edu.cn

E-mail address: cshw45638760yahoo.cn 